\title{
Sec24- and ARFGAP1-Dependent Trafficking of GABA Transporter-1 Is a Prerequisite for Correct Axonal Targeting
}

\author{
Veronika Reiterer, ${ }^{1}$ Susanne Maier, ${ }^{1}$ Harald H. Sitte, ${ }^{1}$ Alexander Kriz, ${ }^{2}$ Markus A. Rüegg, ${ }^{2}$ Hans-Peter Hauri, ${ }^{2}$ \\ Michael Freissmuth, ${ }^{1}$ and Hesso Farhan ${ }^{1}$ \\ ${ }^{1}$ Institute of Pharmacology, Medical University of Vienna, 1090 Vienna, Austria, and 2Biozentrum, University of Basel, 4056 Basel, Switzerland
}

The GABA transporter-1 (GAT1) is a prototypical protein of the synaptic specialization. Export of GAT1 from the endoplasmic reticulum (ER) is contingent on its interaction with the COPII (coatomer protein-II) coat subunit Sec24D. Here we show that silencing all four Sec24 isoforms strongly inhibits transport of GAT1 to the cell surface. In contrast, transport of GAT1-RL/AS, a mutant that is deficient in Sec24D recruitment, was not inhibited, suggesting a nonconventional, COPII-independent pathway. However, ARFGAP1 bound directly to the C terminus of both GAT1-RL/AS and wild-type GAT1. Surface expression of GAT1-RL/AS involved ARFGAP1. GAT1-RL/AS appeared to bypass the ER-Golgi-intermediate compartment, but its pathway to the plasma membrane still involved passage through the Golgi. Thus, the GAT1-RL/AS mutant allowed to test whether COPII-dependent ER-export is required for correct sorting of GAT1 to the axon terminal in neuronal cells. In contrast to wild-type GAT1, GAT1-RL/AS failed to be specifically enriched at the tip of neurite extensions of CAD.a cells (a neuroblastoma cell line that can be differentiated into a neuron-like phenotype) and in the axon terminals of hippocampal neurons. These findings indicate that correct sorting to the axon is contingent on ER export via the COPII machinery and passage through the ER-Golgi-intermediate compartment.

Key words: GABA transporter-1; axonal targeting; ARFGAP1; COPII; Sec24; endoplasmic reticulum export

\section{Introduction}

Neuronal differentiation involves compartmentalized sorting of membrane constituents to generate at least two distinct surfaces, the somatodendritic and the axonal membrane. These surfaces differ substantially in their composition. Nevertheless, expansion of either compartment is thought to be dependent on the secretory pathway, which provides for continuous flow of cargo from the endoplasmic reticulum to the cell surface. The basic components of the secretory pathway have been characterized in considerable detail. Export of proteins from the endoplasmic reticulum (ER) is mediated by COPII-coated vesicles (Lee et al., 2004). Assembly of the COPII coat is initiated by activation of the GTPase Sar1, and the sequential recruitment of Sec23-Sec24 dimers and Sec13-Sec31 heterotetramers to form the inner and outer layer of the COPII coat, respectively. COPII vesicles bud from specialized regions of the ER termed ER exit sites (ERES). In mammalian cells, COPII vesicles fuse with the ER-Golgi intermediate compartment (ERGIC) (Appenzeller-Herzog and Hauri, 2006). In addition, it was shown (in vitro) that COPII vesicles fuse

Received July 22, 2008; revised 0ct. 8, 2008; accepted 0ct. 14, 2008.

This work was supported by grants from the Austrian National Bank (10507) and from the Austrian Science Fund (FWF) P18072 and the FWF-sponsored SFB-35. H.F. is a recipient of a Schrödinger Fellowship from the FWF (J2680). We thank Julie Donaldson and Jennifer Lippincott-Schwartz for generous gifts of plasmids. We thank Dan Cassel for ARFGAP1 expression vectors, for anti-ARFGAP1 antibody, and for his comments on this manuscript.

Correspondence should be addressed to Michael Freissmuth, Institute of Pharmacology, Medical University of Vienna, Waehringer Strasse 13, A-1090 Vienna, Austria. E-mail: michael.freissmuth@meduniwien.ac.at.

V. Reiterer's and H. Farhan's present address: Biozentrum, University of Basel, Klingelbergstrasse 70, 4056 Basel, Switzerland.

DOI:10.1523/JNEUROSCI.3451-08.2008

Copyright $\odot 2008$ Society for Neuroscience $\quad 0270-6474 / 08 / 2812453-12 \$ 15.00 / 0$ homotypically and thus may generate the ERGIC (Xu and Hay, 2004). Anterograde movement of cargo also requires the COPI coat, the assembly of which is initiated by the recruitment of the GTPase ARF1 (Lee et al., 2004).

In neuronal cells, the secretory pathway has features indicative of specialization. Originally, ERGIC-53 [the marker protein of the intermediate compartment (Hauri et al., 2000)] was thought to be confined to the neuronal soma (Krijnse-Locker et al., 1995). However, its presence was subsequently demonstrated in dendritic spines (Pierce et al., 2000). Similarly, Golgi outposts were identified in dendrites (Horton et al., 2005). Mutations have recently been identified, which specifically inhibit dendritic arborization. These included loss of function mutations of Sec23 and Sarl (Ye et al., 2007). Together, these findings are consistent with a specialized role of the secretory pathway in dendritogenesis. In contrast, the generation of axons is apparently COPII independent, because inactivation of genes encoding Sar1, Sec23, and Rab1 or downregulation of Sar1 expression does not affect the axonal length (Ye et al., 2007).

Neurotransmitter transporters terminate synaptic transmission by retrieving released neurotransmitters from the synaptic cleft into the presynaptic specialization. It is evident that transporters have to be specifically targeted to the axonal compartment. However, it is not clear how GAT1 and other related SLC6 family members are enriched in the axonal compartment. ER export of GAT1 requires the formation of homooligomers (Scholze et al., 2002) and the recruitment of Sec24D to a motif comprising ${ }^{566} \mathrm{RL}^{567}$ in its $\mathrm{C}$ terminus (Farhan et al., 2004, 2007). If the Sec24D binding site is mutated, the resulting GAT1-RL/AS fails to undergo COPII-dependent export from the ER, but 
A

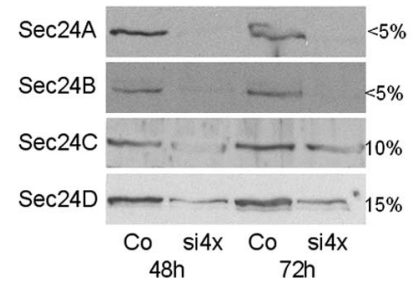

B

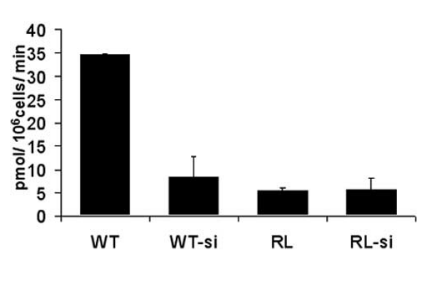

Figure 1. Depletion of Sec24-isoforms reduces the amount of wild-type GAT1 but not of mutant GAT1-RL/AS at the cell surface. $A$, HeLa cells $\left(2 \times 10^{5}\right)$ plated into 6 -well plates were reverse transfected with control siRNA (Co) or with a mix of siRNAs against Sec24A, Sec24B, Sec24C, and Sec24D (si). Total amount of oligonucleotides was the same in both transfections $(260 \mathrm{ng})$. Cells were collected and lysed 48 and $72 \mathrm{~h}$ after transfection, and immunoblots for individual Sec24 isoforms were performed. The numbers on the right side indicate the densitometrically estimated extent (using ImageJ software) of knockdown in percentage of control at $72 \mathrm{~h}$; in all instances $(n=3)$, levels of Sec $24 \mathrm{~A}$ and Sec $24 \mathrm{~B}$ were $\ll 5 \%$; levels of Sec $24 \mathrm{C}$ and Sec $24 D$ were $10 \pm 8.6$ and $15 \pm 5.7 \%$ of control at $72 \mathrm{~h}$ (means \pm SD). $\boldsymbol{B}$, HeLa cells were pretreated with siRNAs as outlined for $\boldsymbol{A}$. After $48 \mathrm{~h}$, cells were transfected with a plasmid encoding either YFP-tagged wild-type GAT1 (WT) or YFP-tagged GAT1-RL/AS (RL). Eight hours thereafter, the content of each well was transferred to seven wells of a 24-well plate. Uptake of $\left[{ }^{3} \mathrm{H}\right] \mathrm{GABA}$ (specific activity $\sim 15 \mathrm{cpm} / \mathrm{pmol}$ ) was performed $24 \mathrm{~h}$ after plasmid transfection. The total GABA concentration was $5.5 \mu \mathrm{m}$. Incubation time was $3 \mathrm{~min}$, and the experiment was performed at room temperature $\left(\sim 24^{\circ} \mathrm{C}\right)$. Nonspecific uptake was measured in the presence of $10 \mu \mathrm{m}$ tiagabine. Data are means $\pm \operatorname{SEM}(n=3)$.

reaches the cell surface via an uncharacterized pathway (Farhan et al., 2007). This mutant provides an opportunity to test whether or not targeting to the axonal compartment depends on the COPII-machinery. Here, we show that trafficking of GAT1RL/AS to the Golgi is COPII independent but involves ARFGAP1. This nonconventional ER exit route, however, precludes correct sorting to the axonal compartment.

\section{Materials and Methods}

Cell culture and transfection. HEK293 cells and HeLa cells were cultured in DMEM supplemented with $10 \%$ fetal bovine serum, L-glutamine, and antibiotics. For microscopy of HEK 293 cells, $3 \times 10^{5}$ cells were seeded on $15 \mathrm{~mm}$ poly-D-lysine-coated coverslips. Transient transfections were done with the $\mathrm{CaPO}_{4}$ precipitation method. HeLa cells were transfected using FuGene (Roche). When we wanted to purify 6 xHis-tagged constructs the transfection of HEK293 cells was performed with Lipofectamine Plus (Invitrogen). LdLF cells were cultured in Ham's F-12 medium supplemented with $5 \%$ fetal bovine serum and antibiotics. For microscopy, $4 \times 10^{5}$ cells were seeded on $15 \mathrm{~mm}$ poly-D-lysine-coated coverslips. Cells were transfected with Lipofectamine Plus according to the manufacturer's instructions. CAD cells were cultured in an 1:1 mix of DMEM and Ham's F-12 medium supplemented with 1\% glutamine, 5\% FCS, and antibiotics. For microscopy, $2.5 \times 10^{5}$ cells were seeded on poly-D-lysine-coated coverslips. CAD cells were transiently transfected using Lipofectamine Plus (Invitrogen). For experiments with siRNA (all from Qiagen), $2 \times 10^{5}$ HeLa cells were reverse transfected with $5 \mathrm{nM}$ siRNA using HiPerFect (Qiagen) according to the manufacturer's instructions.

Antibodies and plasmids. The following antibodies were used: mouse monoclonal antibody against ERGIC-53 (G1/93) and rabbit polyclonal antibody against giantin were from Alexis Corporation, the anti-HA antibody was from Santa Cruz, the anti-His ${ }_{6}$ antibody was from Qiagen. Plasmids encoding for the following constructs were used: HA-tagged ARF1 was a gift from Julie Donaldson (National Institutes of Health, Bethesda, MD), His-tagged ARFGAP1 was a gift from Dan Cassel (Technion, Haifa, Israel), and YFP-tagged ARFGAP1 and $\triangle 64$ ARFGAP1 were gifts from Jennifer Lippincott-Schwartz (National Institutes of Health, Bethesda, MD).

Assay for $\left[{ }^{3} \mathrm{H}\right] G A B A$ uptake. Uptake of $\left[{ }^{3} \mathrm{H}\right] \mathrm{GABA}$ was determined as described in detail previously (Scholze et al., 2002). In brief, transiently
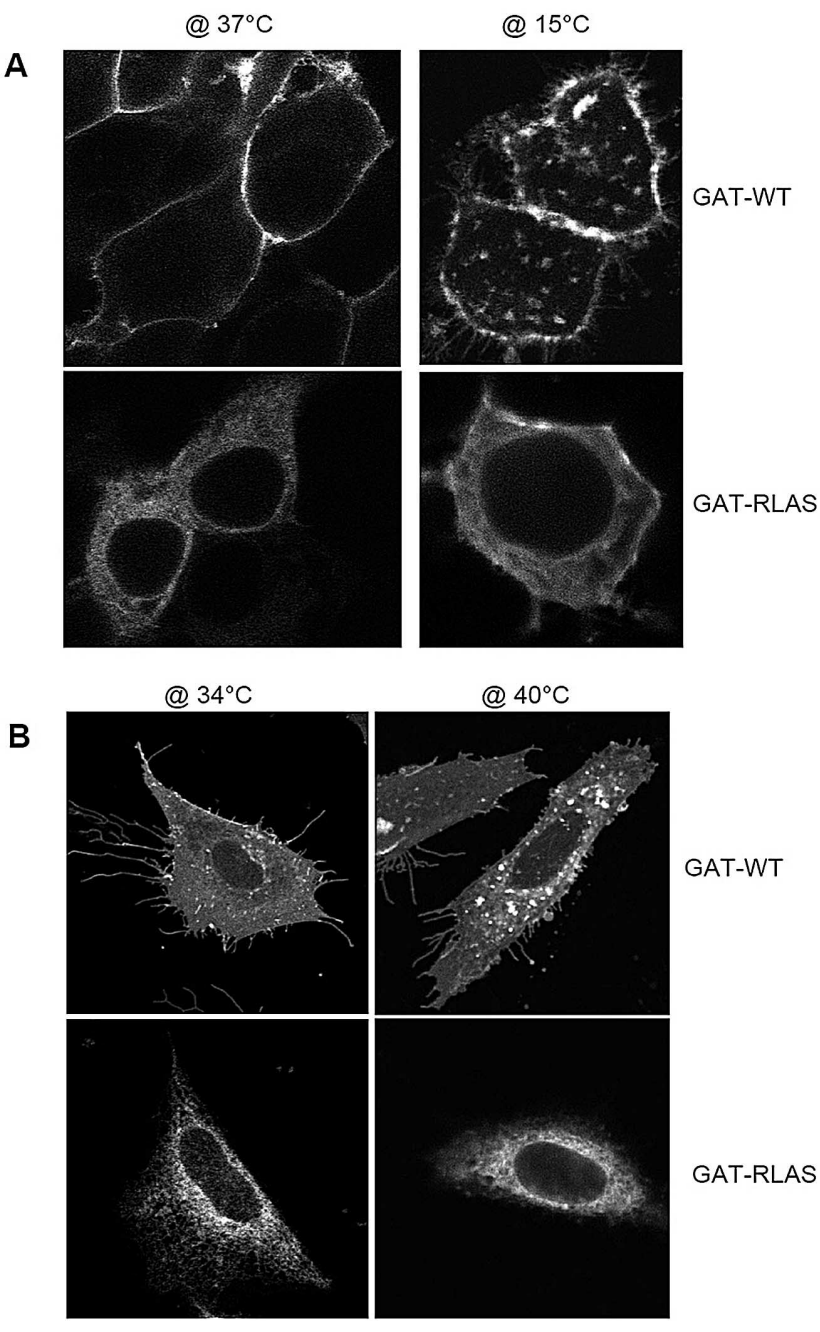

GAT-WT

Figure 2. Effect of temperature on the subcellular distribution of GAT1 and GAT1-RL/AS. $A$, HEK293 cells expressing YFP-tagged GAT1 or YFP-tagged GAT1-RL/AS were kept either at $37^{\circ} \mathrm{C}$ or incubated at $15^{\circ} \mathrm{C}$ for $3 \mathrm{~h}$. Cells were fixed with $4 \%$ paraformaldehyde and imaged subsequently. The number of cells containing fluorescence in punctate structures were scored; these numbers were 0/20 and 16/26 for YFP-tagged GAT1 and 0/20 and 0/18 for YFP-tagged GAT1$\mathrm{RL} / \mathrm{AS}$ at $37^{\circ}$ and $15^{\circ}$, respectively (data from 3 independent experiments). $\boldsymbol{B}$, IdIF cells expressing YFP-tagged GAT1 (top row) or YFP-tagged GAT1-RL/AS were either kept at $34^{\circ} \mathrm{C}$ (permissive temperature) or incubated for $5 \mathrm{~h}$ at $40^{\circ} \mathrm{C}$ (restrictive temperature) and subsequently fixed with $4 \%$ paraformaldehyde. Images were captured by confocal microscopy. The number of cells containing fluorescence in punctate structures were scored: these numbers were $0 / 20$ and 20/20 for YFP-tagged GAT1 and 0/20 and 0/20 for YFP-tagged GAT1-RL/AS at $34^{\circ}$ and $42^{\circ}$, respectively (data from 3 independent experiments).

transfected HEK293 cells $\left(3 \times 10^{5} /\right.$ well $)$ were seeded into 48 well dishes $24 \mathrm{~h}$ after transfection; after $24 \mathrm{~h}$, the cells were incubated in the presence of $20 \mu \mathrm{M}\left[{ }^{3} \mathrm{H}\right] \mathrm{GABA}$ (specific activity $\sim 83 \mathrm{cpm} / \mathrm{pmol}$ ) for $3 \mathrm{~min}$; nonspecific uptake was defined as the amount of cell associated radioactivity determined in the presence of $100 \mu \mathrm{M}$ tiagabine.

Confocal fluorescence microscopy and FRAP. Fluorescence images were acquired with a $40 \times$ oil-immersion objective with a Zeiss LSM510 confocal microscope and digitally captured. The images were converted to TIF files and analyzed using the ImagePro Plus software (Media Cybernetics). The colored images were converted to 12-bit gray scale images. Under these conditions the software recognizes 4095 potential gray values. The contrast was enhanced by excluding the lower $20 \%$ of the gray scale. For quantifying the accumulation of proteins in the ERGIC and their colocalization (see Figs. 3, 11), all bright objects that were between 1.5 and 10 pixels were defined as peripheral ERGIC structures. The same procedure was used to estimate the extent of colocalization in neurons, 

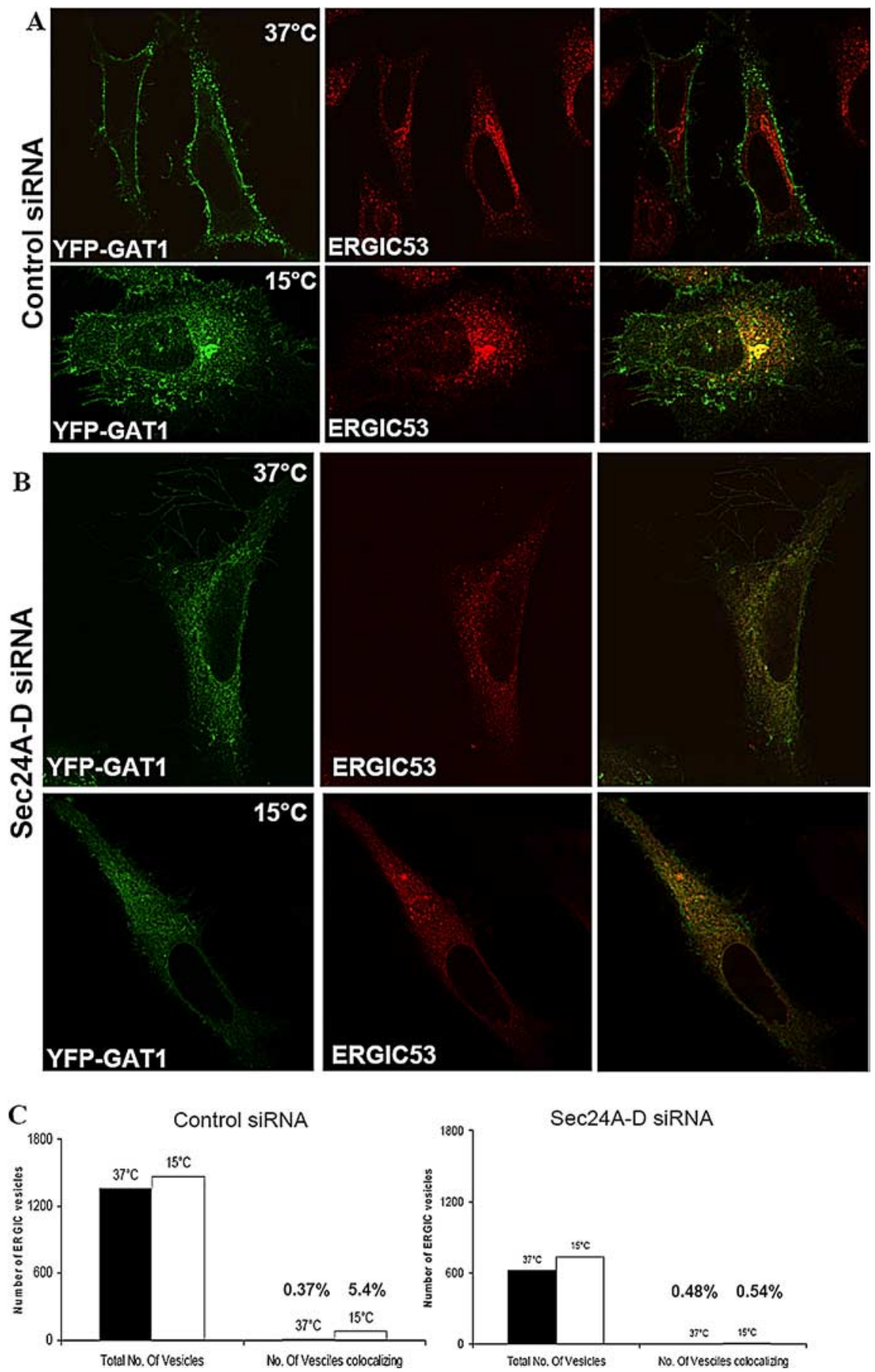

Figure 3. Effect of temperature on localization of GAT1 in the ERGIC in the presence or absence of Sec24. HeLa cells were transfected with a control siRNA $(\boldsymbol{A})$ or siRNAs against Sec24A-D $(\boldsymbol{B})$. The total amount of siRNA and the amount of transfection reagent was the same. After $48 \mathrm{~h}$, cells were transfected with plasmids encoding YFP-tagged GAT1. On the next day, cells were either fixed directly (top rows in $\boldsymbol{A}$ and $\boldsymbol{B}$ ) or after an incubation for $3 \mathrm{~h}$ at $15^{\circ} \mathrm{C}$ (bottom rows in $\boldsymbol{A}$ and $\boldsymbol{B}$ ) followed by immunostaining for ERGIC-53. Images were acquired with a confocal microscope. C, The total number of ERGIC vesicles was quantified by counting the ERGIC-53 positive structures using ImagePro Plus software as outlined in Materials and Methods. The number of ERGIC53 vesicles that contained YFP-GAT1 was also quantified in the same cells; numbers above the bars indicate the percentage of vesicles containing YFP-GAT1. Data are from three independent transfections.

where larger visual field were examined, except that no upper limit was imposed, i.e., structures larger than 1.5 pixels were quantified. Fluorescence recovery after photobleaching (FRAP) was also recorded on a Zeiss LSM510 confocal laser scanning microscope. Transfected cells were examined with an oil-immersion objective (40-fold magnification). Circular regions of interest were specified for bleaching and scanning (bleach regions). Before bleaching, a scan was obtained; the area of interest was then bleached with 70 iterations at maximum laser power $(30 \mathrm{~mW}$, at $514 \mathrm{~nm})$. Subsequently, pictures were captured (40 scans in $40 \mathrm{~s}$ ) with $\sim 4 \%$ of laser power. The recorded fluorescence intensities were digitized and averaged over the bleach region using ScionImage 4.0.2; these values were plotted and subjected to nonlinear curve fitting to the equation describing the monoexponential rise to a maximum: $y=$ bottom + (top - bottom $) \times\left(1-e^{-k x}\right)$, where "bottom" refers to the relative fluorescence intensity immediately after bleaching (before recovery) and "top" to the value that is reached at equilibrium (after recovery). Imaging of neurons was performed with a LeicaSPE confocal laser scanning microscope using the $40 \times$ oilimmersion objective.

Purification of GST-tagged proteins, GSTpulldown and purification, and His ${ }_{6}$-tagged ARFGAP1 and ARF. Purification of GST-tagged proteins and GST pulldown have been described in detail previously (Farhan et al., 2007). For purification of $\mathrm{His}_{6}$-ARF1 and $\mathrm{His}_{6}-$ ARFGAP1, HEK293 cells were transfected with plasmids encoding either $\mathrm{His}_{6}$-ARF1 or $\mathrm{His}_{6}{ }^{-}$ ARFGAP1. The transfection was performed with Lipofectamine Plus (Invitrogen). Twentyfour hours after transfection, cells were scrapped down and lysed in lysis buffer (300 mм NaCl, 50 mм NaH${ }_{2} \mathrm{PO}_{4}, 10 \mathrm{~mm}$ imidazole, $\mathrm{pH}$ adjusted to 7.5 with $\mathrm{HCl}$, and $0.05 \%$ Tween $20)$ and then sonicated 4 times in a water bath for $10 \mathrm{~s}$. The reaction was then centrifuged at $10,000 \times g$ for $10 \mathrm{~min}$. The supernatant was incubated with Ni-NTA Magnetic Agarose beads (Qiagen) for $90 \mathrm{~min}$. Beads were washed twice in wash buffer $(300 \mathrm{~mm} \mathrm{NaCl}, 50 \mathrm{~mm}$ $\mathrm{NaH}_{2} \mathrm{PO}_{4}, 20$ mm imidazole, $\mathrm{pH}$ adjusted to 7.5 with $\mathrm{HCl}$, and $0.05 \%$ Tween 20 ). The protein was eluted in elution buffer $(300 \mathrm{~mm} \mathrm{NaCl}, 50$ $\mathrm{mm} \mathrm{NaH} \mathrm{N}_{2} \mathrm{PO} 4,250 \mathrm{~mm}$ imidazole, $\mathrm{pH}$ adjusted to 7.5 with $\mathrm{HCl}$, and $0.05 \%$ Tween 20 ).

Preparation of rat hippocampal neurons. High-density cultures $\left(\sim 5 \times 10^{4}\right.$ cells $\left./ \mathrm{cm}^{2}\right)$ were established from 18-d-old Wistar rat hippocampi. The fetal hippocampi were removed from the brain, washed once in HBSS, and trypsinized for $15 \mathrm{~min}$ at $37^{\circ} \mathrm{C}$. Thereafter, hippocampi were washed again in HBSS followed by additional washes in prewarmed plating medium (DMEM/Glutamax, 0.6\% glucose, 10\% fetal calf serum, and antibiotics). Finally, the hippocampi were dissociated in the plating medium on glass coverslips (precoated with polyL-lysine; $1 \mathrm{mg} / \mathrm{ml}$ in borate buffer, $\mathrm{pH} 8.5$; coated overnight and washed twice with water). After $3 \mathrm{~h}$ from the initial seeding the medium was changed to Neurobasal medium (Invitrogen) supplemented with $0.5 \mathrm{~mm}$ glutamine, antibiotics, and B27 supplement (Invitrogen). Neurons were transfected after $7 \mathrm{~d}$ in vitro using Lipofectamine 2000 (Invitrogen) according to the manufacturer's instructions.

Immunoprecipitation experiments. Brains from adult rats (cerebrum, cerebellum, and medulla oblongata) were homogenized in lysis buffer (50 mu Tris. $\mathrm{HCl}, \mathrm{pH} 7.4,100 \mathrm{~mm} \mathrm{NaCl}, 1 \%$ Triton X-100) supplemented with protease inhibitors. The homogenate was centrifuged at $10,000 \times g$ for $10 \mathrm{~min}$ at $4^{\circ} \mathrm{C}$. The supernatant was recentrifuged at 25,000 $\times \mathrm{g}$ for additional $10 \mathrm{~min}$ at $4^{\circ} \mathrm{C}$. Anti-GAT1 antibody (ab426 from Abcam) was 
added to the supernatant $(1 \mathrm{ml})$, and the sample was rotated overnight at $4^{\circ} \mathrm{C}$. On the next day protein $\mathrm{G}$ beads ( $50 \mu$ l of 1:1 slurry) were added for additional $3 \mathrm{~h}$. After washing the beads three times in lysis buffer, proteins were released by boiling for $5 \mathrm{~min}$ in sample buffer. The eluted proteins were subjected to SDS-PAGE.

\section{Results}

COPII depletion does not affect trafficking of GAT1-RL/AS to the plasma membrane.

Our previous observation that GAT1$\mathrm{RL} / \mathrm{AS}$ is unable to bind to Sec24D (Farhan et al., 2007) raises the possibility that its exocytic trafficking occurs in a COPIIindependent manner. Alternatively, GAT1-RL/AS may be incorporated by default into COPII vesicles. To test for COPII dependence, we performed a quadruple knockdown of all Sec24 isoforms (Wendeler et al., 2007) and estimated the presumptive surface expression of GAT1 by $\left[{ }^{3} \mathrm{H}\right] \mathrm{GABA}$ uptake. We chose HeLa cells because of their efficient uptake of siRNA (Wendeler et al., 2007). Sec24 isoforms were efficiently knocked down (Fig. 1A). GAT1 was transfected $48 \mathrm{~h}$ after knockdown, and $\left[{ }^{3} \mathrm{H}\right] \mathrm{GABA}$ uptake was measured $72 \mathrm{~h}$ after knockdown. Of note, Sec24 levels were strongly reduced already after $48 \mathrm{~h}$, indicating that when these cells started to express GAT1, they already had low levels of Sec 24 which continued to be low to the day of the uptake experiment. As expected, the quadruple knockdown of Sec24 isoforms strongly reduced surface expression of wild-type GAT1 down to a level comparable to GAT1-RL/AS (Fig. $1 B)$. In contrast, the level of GAT1-RL/AS surface expression was not affected by the knockdown.

Given the inability of GAT1-RL/AS to interact with Sec24 and the strongly reduced cellular Sec24 levels, it becomes very unlikely that anterograde trafficking of GAT1-RL/AS is COPII dependent. It rather appears that the mutant exits by another mechanism, which, for the lack of better understanding, we will refer to as a nonconventional, default pathway.

\section{GAT1-RL/AS cannot be trapped in the ERGIC}

It was of interest to understand the route taken by vesicles subject to this nonconventional ER exit, specifically whether the vesicles also reach the ERGIC. Using an in vitro system, it was shown that COPII vesicles homotypically fuse ( $\mathrm{Xu}$ and Hay, 2004). This was proposed to be a mechanism for de novo formation of the ERGIC (Xu and Hay, 2004). Alternatively, COPII vesicles may fuse heterotypically with the ERGIC. In any case trafficking of a protein through the ERGIC requires it to be incorporated into COPII vesicles.

Many secretory proteins can be trapped in the ERGIC by lowering the temperature to $15^{\circ} \mathrm{C}$ (Saraste and Kuismanen, 1984, Schweizer et al., 1990). Incubation of HEK 293 cells at $15^{\circ} \mathrm{C}$ for $3 \mathrm{~h}$ caused retention of wild-type GAT1 in punctate structures that stained for ERGIC-53 (Fig. $2 \mathrm{~A}$ ). In contrast, the distribution pattern of GAT1-RL/AS was not affected by low temperature (Fig. $2 A$ ). An alternative way to arrest protein transport in the ERGIC is to inactivate COPI function (Pepperkok et al., 1993). This condition can be probed with ldlF cells which express a temperaturesensitive mutant of $\varepsilon$-COP that misfolds at the elevated temperature of $40^{\circ} \mathrm{C}$ (restricted temperature) and thereby bocks COPI function (Guo et al., 1994). Protein transport at $34^{\circ} \mathrm{C}$ (permissive temperature) is normal in ldlF cells. At the permissive temperature, wild-type GAT1 was transported to the plasma membrane, whereas GAT1-RL/AS was predominantly in the ER (Fig. $2 B$ ). When cells were incubated at $40^{\circ} \mathrm{C}$ for $4 \mathrm{~h}$, wild-type GAT1 accumulated in punctate structures (Fig. $2 \mathrm{~B}$ ) that stained for ERGIC-53 (data not shown). In contrast, the temperature shift did not affect the distribution of GAT1-RL/AS (Fig. $2 \mathrm{~B}$, bottom row). Thus, the nonconventional route taken by GAT1-RL/AS is most readily explained by a transport pathway that bypasses the ERGIC. 

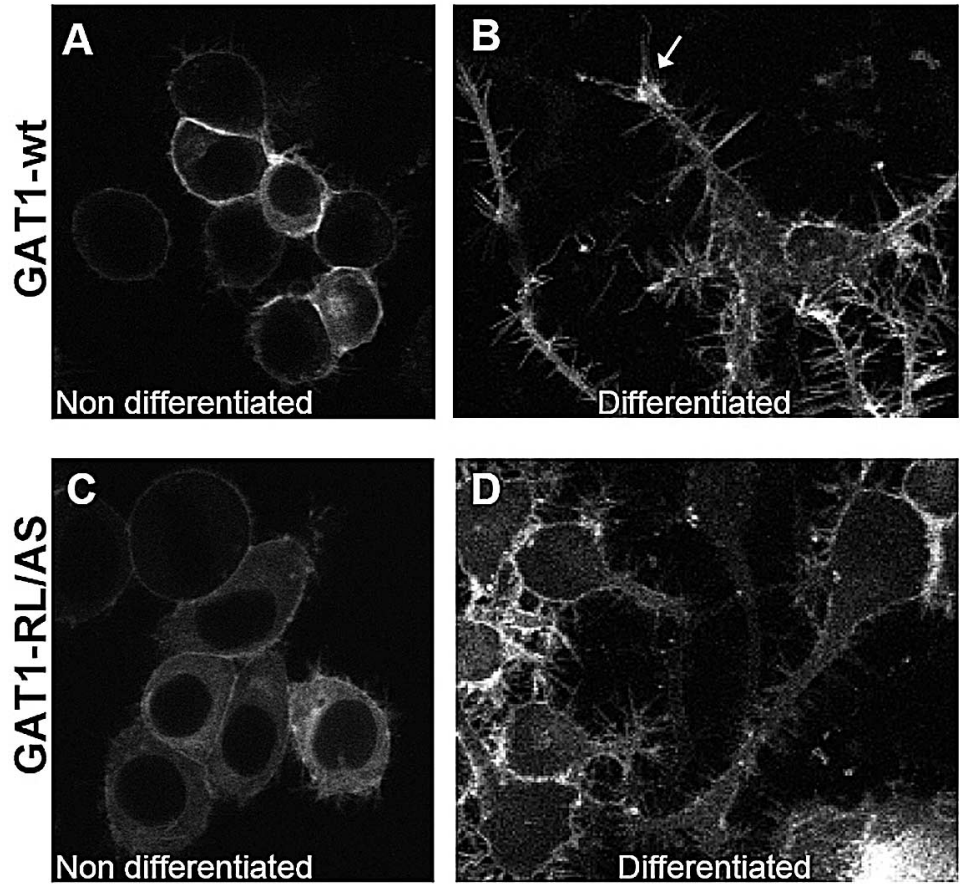

Figure 5. Localization of GAT1 and GAT1-RL/AS in CAD cells. CAD cells were transfected with plasmids encoding YFP-tagged GAT1 $(\boldsymbol{A}, \boldsymbol{B})$ or YFP-tagged GAT1-RL/AS $(\boldsymbol{C}, \boldsymbol{D})$. Cells were either kept in the presence of serum to inhibit differentiation $(\boldsymbol{A}, \boldsymbol{C})$ or in a serum-free medium to induce differentiation $(\boldsymbol{B}, \boldsymbol{D})$ for $24 \mathrm{~h}$. Afterward, cells were fixed, and the distribution of GAT1 was visualized using a confocal microscope. Data were reproduced in two independent experiments; a total of 50 cells were scored (for details, see Results).

If GAT1-RL/AS was not concentrated in the ERGIC because of its inability to bind Sec24, it should in principle be possible to mimic the situation for wild-type GAT1 by depletion of Sec24. We tested this prediction by silencing all four Sec24 isoforms. Accumulation of GAT1 in the ERGIC was assessed by colocalization with ERGIC-53. In cells transfected with the control siRNA, wild-type GAT1 was seen at the cell surface (Fig. $3 A$, top row). If cells were incubated at $15^{\circ} \mathrm{C}$ for $3 \mathrm{~h}, \mathrm{GAT} 1$ was found in punctate ERGIC-53 positive structures (Fig. 3A, bottom row). After Sec24 silencing ERGIC-53 showed an ER pattern and only few punctate structures were seen (Fig. 3B, top row, middle image). Although GAT1 was predominantly retained in the ER in these cells, a fraction of it was still transported to the plasma membrane (Fig. $3 B$, top row, leftmost image). The distribution pattern of GAT1 did not change, if cells deficient in Sec 24 isoforms were placed at $15^{\circ} \mathrm{C}$ for $3 \mathrm{~h}$ (Fig. $3 \mathrm{~B}$ ). Most importantly, the occasional ERGIC53-containing punctate structures were devoid of GAT1 (overlay in bottom right panel of Fig. $3 B$ ). These results suggest that a fraction of GAT1 can take the alternative, nonconventional route to the plasma membrane in cells in which Sec24 is silenced.

\section{The pathway of GAT1-RL/AS involves the Golgi}

Does GAT1-RL/AS pass through the Golgi on its way to the cell surface? We addressed this question by imposing a $20^{\circ} \mathrm{C}$ temperature block known to inhibit exocytic traffic from the Golgi (Griffiths et al., 1985). HEK293 cells were cotransfected with a plasmid encoding YFP-Golgi together with either CFP-tagged wild-type GAT1 or CFP-tagged GAT1-RL/AS. On the following day, the cells were incubated at $20^{\circ} \mathrm{C}$ for $5 \mathrm{~h}$. Both proteins were found to be retained in the Golgi under these conditions (Fig. 4A,B). The data shown in Figure 3 suggested that, in the absence of all Sec24 isoforms, a fraction of wild-type GAT1 also used the nonconven- tional default pathway. We analyzed whether GAT1 reaches the Golgi in Sec24silenced cells. Figure 4, $C$ and $D$, shows that GAT1 indeed reaches the Golgi under these conditions at $20^{\circ} \mathrm{C}$. We conclude that the nonconventional default pathway involves the Golgi.

\section{Trafficking of wild-type GAT1 and GAT1-RL/AS in neuronal extensions of CAD cells}

GAT1 is enriched in the axonal compartment (Chiu et al., 2002). It is not clear, however, whether early steps in protein trafficking play a role in determining sorting and targeting of GAT1. There is circumstantial evidence that this occurs early in the secretory pathway. The exocyst, for instance, is required at the very last step (i.e., insertion into the plasma membrane), but disruption of the exocyst traps GAT1 in the ER rather than in a submembrane compartment (Farhan et al., 2004). Factors that determine dendritic sorting of the NMDA receptor also interact with the receptor already at the level of the ER (Sans et al., 2005). The ERGIC has been shown to move into the neurite extensions upon differentiation of PC12 cells (Sannerud et al., 2006). Because GAT1 is also enriched in the neurite extensions of PC12 cells (Farhan et al., 2004), we hypothesized that the physiological trafficking route of GAT1 (i.e., ER $\rightarrow$ ERGIC $\rightarrow$ Golgi) may be important for correct sorting. Any deviation from this pathway (e.g., bypass of the ERGIC) would lead to misrouting of GAT1. The mutant GAT1-RL/AS, which bypasses the ERGIC, provided an opportunity to test this hypothesis in CAD cells, a mouse CNS catecholaminergic cell line derived from the Cath.a tumor cell line (Qi et al., 1997). The rationale for using these cells was that they are readily propagated, can be transfected at a high efficiency and undergo differentiation upon serum removal to develop neurite extensions that express markers of axons and dendrites (Qi et al., 1997; Li et al., 2007). CAD cells were transfected with plasmids encoding YFP-tagged GAT1 or GAT1-RL/AS and the cells were differentiated for $24 \mathrm{~h}$. In nondifferentiated cells the distribution pattern of the two proteins was similar to that in other cell lines, with wild-type GAT1 predominantly visualized at the plasma membrane and GAT1-RL/AS largely retained within the cells (Fig. $5 A, C$ ). Upon induction of differentiation wild-type GAT1 was found in the plasma membrane and was enriched at the tip of the neurites (Fig. 5B, arrow). This was seen in 42 of 50 differentiated cells when scored (on coded prints) by an observer blinded to the nature of the expressed version of GAT1. Due to the prolonged incubation associated with differentiation, GAT1RL/AS escaped to the cell surface. In contrast to wild-type GAT1, however, it was not specifically enriched at the tip of neurite extensions but homogeneously distributed over cell bodies and neurite extensions (Fig. 5D). When quantified by an unbiased observer, enrichment at the tip of the neurite extension was only scored in 6 of 50 cells expressing GAT1-RL/AS.

We compared the mobility of GAT1 molecules that resided in the plasma membrane compartment to those delivered to neurite extensions. If GAT1 was enriched in the neurite extensions by 
active targeting/sorting and specific retention, it was predicted to be less mobile. We verified this conjecture by fluorescence recovery after photobleaching (FRAP) experiments: CAD cells were transfected with plasmids encoding YFP-tagged GAT1 and cells were differentiated by serum withdrawal for $24 \mathrm{~h}$. Regions of interest were selected over the neurite (Fig. 6A) and over the cell body (Fig. 6B) and bleached. The intensely fluorescent accumulations in the neurite extensions did not recover over the time course of the experiment (Fig. $6 A, B$ ). In addition, the mobile fraction was higher in somatic regions than in neurites and this difference was statistically significant (Fig. 6C) (21.2 $\pm 3.9 \%$ versus $32.2 \pm 4.2 \%$ - mean $\pm \mathrm{SD} ; n=3$; $p=0.028$ - for neurite and somatic region, respectively). Based on these findings, we conclude that GAT1 is specifically sorted to the neurite extensions to accumulate in specific areas such that it cannot exchange rapidly with the pool of transporter in the other surface membrane compartments.

\section{The RL/AS-mutation acts as a second site suppressor for an ERGIC-trapped GAT1 mutant}

If the tri-hydrophobic motif ${ }^{569} \mathrm{VMI}^{571}$ in the $\mathrm{C}$ terminus of GAT1 is replaced by three serines, the resulting GAT1-SSS undergoes COPII-dependent export but becomes trapped in the ERGIC and does not reach the cell surface (Farhan et al., 2008). This was also recapitulated in CAD cells (Fig. 7A): we coexpressed CFP-tagged GAT1-SSS and YFP-tagged serotonin transporter (SERT). The latter was used to unequivocally visualize the cell surface and in particular the sprouting neuronal extensions and spikes. It is evident from Figure 7A that GAT1-SSS remained in punctate structures, while the serotonin transporter resided at the cell surface and was enriched on filopodia-like projections arising from the developing neurite extensions. In all cells examined (including neurons), GAT1-SSS is trapped in the ERGIC after undergoing (COPII-dependent) ER export (Farhan et al., 2008). Because the RL/AS mutation prevents COPII recruitment and thus COPII-dependent export, we surmised that a mutation of the RL-motif should act as a second site suppressor within the GAT1-SSS background. This was the case: while the bulk of the double mutant GAT1-RL/AS-SSS was diffusely distributed within the cell, a fraction of this transporter did escape to the cell surface (Fig. $7 B$, middle panel). However, in contrast to the coexpressed SERT (Fig. 7B, top panel), GAT1-RL/AS-SSS was not enriched at the tips of the growing neurite extensions (marked by arrowhead, in Fig. $7 B$ ), where the green pseudocolor representing SERT was dominant in the overlay (Fig. $7 B$, bottom panel).

\section{Lack of axonal enrichment of GAT1-RL/AS in hippocampal neurons}

While neuroblastoma and related cells can differentiate into a phenotype resembling catecholaminergic neurons, the nature of their neurite extensions is difficult to assess; in fact, their neuritic extensions contain markers of both axons and dendrites (Li et al., 2007). Therefore, we investigated trafficking of wild-type GAT1 and GAT1-RL/AS in rat hippocampal neurons prepared from embryonic rats (E17). After $7 \mathrm{~d}$ in culture neurons were transfected with the indicated plasmids and fixed after $48 \mathrm{~h}$. Immunofluorescent labeling of MAP2 was performed to stain dendrites. Processes that were negative for MAP2 were considered axons. While wild-type GAT1 nicely decorated the plasma membrane (Fig. 8 A), GAT1-RL/AS was found predominantly in the ER and a minor fraction of it was found at the plasma membrane (Fig. $8 B$ ). The axons terminal is the site of most active membrane addition. When we inspected axons we found that wild-type GAT1 was strongly enriched in the axon terminal (Fig. $8 C$ ), while GAT1-RL/AS was not (Fig. $8 D$ ). To quantify the magnitude of this differential enrichment, we compared the fluorescence intensity of the axon terminal with the axon which is immediately adjacent to it. The measured fluorescence intensity was divided by the area to extract the density of YFP molecules (i.e., GAT1 or its mutant). Because we observed that neuronal processes run through several focus planes, we measured the density of fluorescent molecules in 4 different confocal planes, each separated 200 $\mathrm{nm}$ from the next. Using this approach we found that the density of wild-type GAT1 was 2-fold higher in the axon terminal than in more proximal parts (Fig. $8 E$ ). No such difference was observed with GAT1-RL/AS (Fig. $8 F$ ). 

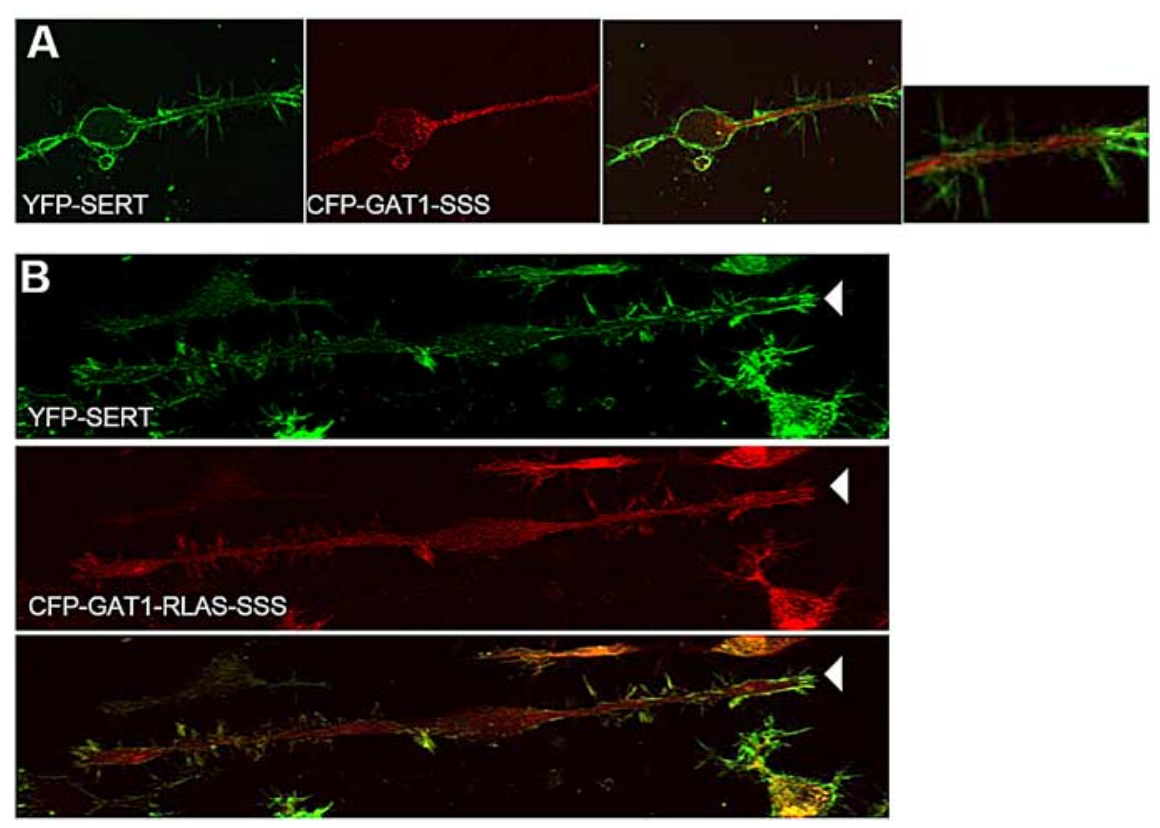

Figure 7. Colocalization of GAT1 mutants and SERT in differentiated CAD cells. CAD cells were transfected with plasmids encoding YFP-tagged SERT and CFP-tagged GAT1 mutants GAT1-SSS ( $\boldsymbol{A}$ ) and GAT1-RL/AS-SSS ( $\boldsymbol{B})$. After $24 \mathrm{~h}$, differentiation was induced by serum withdrawal for a period of $18-24 \mathrm{~h}$. Thereafter, CFP and YFP images of live cells were acquired by confocal microscopy as indicated and overlaid (right panel in $\boldsymbol{A}$, bottom panel in $\boldsymbol{B}$ ). The blow-up in $\boldsymbol{A}$ highlights the punctate accumulation of CFP-tagged GAT1-SSS and the fact that this mutant fails to reach the cell surface. The arrowhead in $\boldsymbol{B}$ highlights the tips of a neurite extension, where YFP-SERT, but not CFP-GAT1-RL/AS-SSS, is enriched. Data are representative of four independent transfections with at least 10 visual fields examined/transfection.

Finally, we coexpressed a dominant-negative version of Sar1 (Sar1-T39N) together with wild-type GAT1 in hippocampal neurons (DIV 9). We chose to use Sar1-T39N instead of knockdown of Sec24 isoforms to interfere with COPII function because it is very difficult to reliably achieve a quadruple knockdown in primary neurons. Sar1-T39N is locked in the GDP-bound state and exerts a dominant-negative effect by sequestering Sec12, its cognate (ER-resident) exchange factor. The Sar1-plasmid was used in 2:1 excess over the GAT1 plasmid to increase the probability that a GAT1-expressing neuron also expressed Sar1-T39N. As shown in Figure 9, GAT1 was predominantly visualized within the cell, which is indicative of a disturbed COPII function. Importantly, under this condition we failed to detect GAT1 in a MAP2-negative neurite (= axon) (cf. Fig. 9 and Fig. 8).

\section{ARFGAP1 interacts with the C terminus of GAT1}

The data above clearly showed that COPII-dependent ER export and transit through the ERGIC were essential for correct axonal targeting. If our interpretation were correct, axonal targeting also ought to be impaired upon inhibition of exit from the ERGIC. Accordingly, we searched for a candidate protein that controlled exit of GAT1 from the ERGIC based on the following rationale: although protein transport between ER and Golgi is COPII dependent, various studies indicate that COPI might also play a role (Pepperkok et al., 1993; Gomez et al., 2000). Recently, ARFGAP1 was proposed to serve as a cargo adaptor that mediates incorporation of proteins into COPI vesicles (Lee et al., 2005). We surmised that ARFGAP1 also regulates trafficking of GAT1. If so, GAT1 can be expected to directly bind to ARFGAP1. This was indeed the case. Purified ARFGAP1 interacted with a fusion protein comprising the $\mathrm{C}$ terminus of GAT1 fused to GST (Fig. 10A). Interestingly, however, the interaction with ARFGAP1 was not affected by the RL/AS mutation that disrupts binding to the COPII subunit Sec24D (Fig. 10A).

The eponymous role of ARFGAP1 is to deactivate Arf1 by promoting GTP hydrolysis. Deletion of the N-terminal 64 aa of ARFGAP1 ( $\Delta 64$-ARFGAP1) renders the protein catalytically inactive (Huber et al., 1998). To test whether the catalytic domain of ARFGAP1 is required for its binding to GAT1, we expressed an YFP-tagged version of $\triangle 64$-ARFGAP1 in HEK293 cells and prepared cytosol from these cells. The cytosol was incubated with the fusion protein comprising GST and the GAT1 C terminus and a GST-pulldown experiment was performed. Figure $10 \mathrm{~B}$ shows that $\Delta 64$-ARFGAP1 interacted with both the C terminus of wild-type GAT1 and GAT1RL/AS. Thus, the catalytic domain of ARFGAP1 is not required for interaction with the GAT1 C terminus.

\section{Interactions of Arf1 and the GAT1 C terminus with ARFGAP1 are mutually exclusive}

It appears counterintuitive that ARFGAP1 does both, inactivation of Arf1 and sorting of cargo. To address this issue we tested binding of purified $\mathrm{His}_{6}$-ARFGAP1 to the $\mathrm{C}$ terminus of GAT1 alone or after it had been preincubated with purified $\mathrm{His}_{6}$-Arfl in the presence of aluminum, magnesium, and fluoride. In the presence of $\mathrm{Mg}^{2+}$, $\mathrm{AlF}_{4}$ (or $\mathrm{AlF}_{3}$ ) can bind to GDP-liganded G-proteins and mimic the transition state of GTP hydrolysis provided that this transition state is stabilized by an arginine finger (Scheffzek et al., 1997). In the ARFGAP1-Arf1 complex, the arginine finger is provided in trans by the GAP domain. Preloading of ARFGAP1 with Arfl in the presence of $\mathrm{Mg}^{2+}$ and $\mathrm{AlF}_{3}$ precluded the interaction with C terminus of GAT1 (Fig. 10C, first and second lane). This suggested that the $\mathrm{C}$ terminus of GAT1 and Arf1 bound to ARFGAP1 in a mutually exclusive manner. If this was the case, preincubation of ARFGAP1 with the C terminus of GAT1 ought to blunt its interaction with Arfl. We verified this prediction by preincubating purified $\mathrm{His}_{6}$-ARFGAP1 with the GST-tagged C terminus of GAT1 (or vehicle as a control). This incubation mimics the state where ARFGAP1 is in its putative "cargo sorting" conformation. Subsequently, the proteins were incubated with cytosol prepared from HEK293 cells overexpressing HA-tagged Arfl in the presence of $\mathrm{Mg}^{2+}$ and $\mathrm{AlF}_{3}$. $\mathrm{His}_{6}$-ARFGAP1 was retrieved with Nickel NTA-beads and the amount of immobilized HA-tagged Arf1 was quantified by immunoblotting (Fig. 10D). Preincubation of ARFGAP1 with the C terminus of GAT1 reduced its interaction with Arf1 (Fig. 10D, cf. middle and left lane). We ruled out that the interaction with GST-tagged GAT1 C terminus masked the hexa-histidine tag and thus interfered with immobilization of ARFGAP1 on Nickel NTA-beads: Comparable amounts of $\mathrm{His}_{6}$-ARFGAP1 were bound to the Nickel NTAbeads in the absence and presence of the GST-tagged C terminus of GAT1 (Fig. $10 \mathrm{D}$, bottom row). In addition, similar results were obtained, if ARFGAP1 was preincubated with the $\mathrm{C}$ terminus proper (after it had been cleaved from the GST by Factor Xa; data not shown). These observations are consistent with a model 
where ARFGAP1 interacts directly with the cargo GAT1 and where this interaction precedes the deactivation of Arf1.

\section{Interaction of GAT1 and ARFGAP1 in native tissue}

The bulk of GAT1 in the brain resides at the plasma membrane, but there must be a fraction of the protein that, at steady state, is in the secretory pathway to account for the rate of protein turnover. This portion of GAT1 must be associated with components of the secretory machinery including ARFGAP1, if this interaction was relevant in vivo. We therefore prepared lysates from adult rat brain and immunoprecipitated GAT1 (Fig. 10 E, middle row). Sec24D was found in a complex with GAT1 (Fig. $10 \mathrm{E}$, top row) as predicted from our previous work (Farhan et al., 2004, 2007). In addition and importantly, ARFGAP1 was also coimmunoprecipitated in a complex with GAT1 (Fig. 10 E, bottom row). This shows that the interaction between GAT1 and ARFGAP1 (and Sec24D) does take place in the brain and therefore is most likely of physiological relevance.

\section{ARFGAP1 controls trafficking of wild-type GAT1}

Incorporation of cargo into COPI vesicles is strictly dependent on GTP hydrolysis (Nickel et al., 1998; Pepperkok et al., 2000). The above results suggested a role for ARFGAP1 in trafficking of GAT1. Since ERGIC to Golgi transport requires COPI we expect $\Delta 64$-ARFGAP1 to arrest trafficking of wild-type GAT1 in the ERGIC. We first tested whether $\Delta 64$ ARFGAP1 is associated with the ERGIC. Cells were transfected with YFP-tagged $\triangle 64$-ARFGAP1 and stained for ERGIC53. $\Delta 64$-ARFGAP1 was found in punctate structures that colocalized with ERGIC-53 (Fig. 11A). Coexpression of YFP-tagged $\Delta 64$-ARFGAP1 with the CFP-tagged wildtype GAT1 led to retention of the latter in punctate structures which contained both proteins (Fig. $11 \mathrm{~B}$ ). Membrane recruitment of ARFGAP1 can be blocked by incubating cells in the presence of IC261, a casein kinase inhibitor (Yu and Roth, 2002). When HEK293 cells were treated with IC261 for $2 \mathrm{~h}$, GAT1 accumulated in punctate structures that costained for ERGIC-53 (Fig. 11C). Together, these results suggest that ARFGAP1 is required for exit of wild-type GAT1 from the ERGIC.
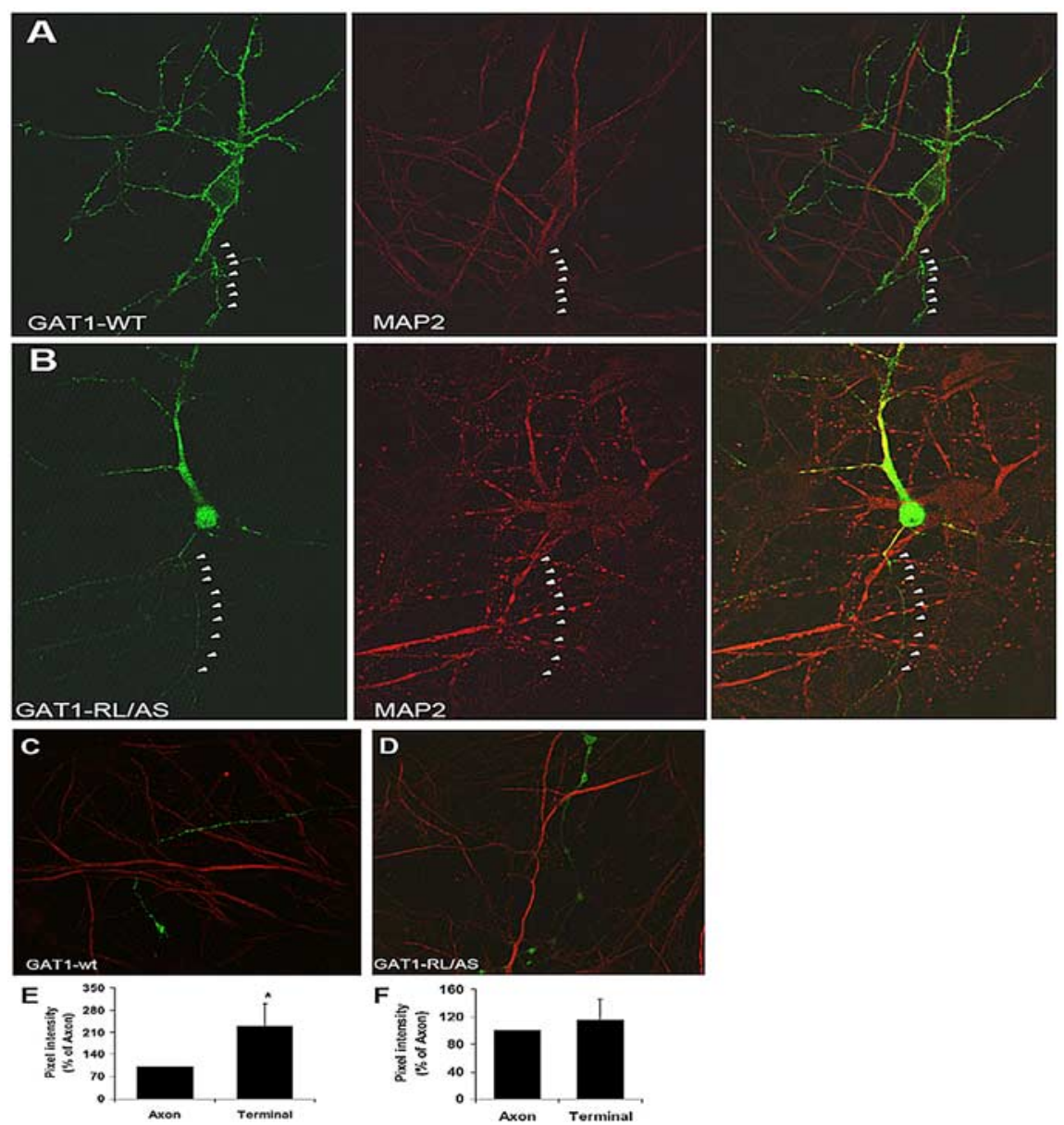

Figure 8. Localization of GAT1 and GAT1-RL/AS in hippocampal neurons. Hippocampal neurons were prepared from rat embryos (E17). After $7 \mathrm{~d}$ in culture, neurons were transfected with plasmids encoding YFP-tagged GAT1 (A, $\boldsymbol{C}$ ) or YFP-tagged GAT1-RL/AS $(\boldsymbol{B}, \boldsymbol{D})$. Cells were fixed $48 \mathrm{~h}$ after transfection, and images were acquired using a confocal microscope. $\boldsymbol{A}$ and $\boldsymbol{B}$ show the neuronal soma with dendrites and the axon which is indicated by white arrowheads. $C$ and $\boldsymbol{D}$ show an axon with its terminal containing wild-type GAT1 or GAT1-RL/AS, respectively. $\boldsymbol{E}$ and $\boldsymbol{F}$ show an evaluation of fluorescence intensity of the axon (an area adjacent to the terminal) versus the axon terminal in neurons expressing wild-type GAT1 (E) or GAT1-RL/AS $(\boldsymbol{F})$. The fluorescence was recorded in four confocal planes separated by $200 \mathrm{~nm}$. The fluorescence intensities of these four individual confocal planes were averaged and treated as a single determination. This analysis was performed for 6 and 5 neurons expressing wild-type GAT 1 and GAT1-RL/AS, respectively. To account for variability in pixel intensity between individual neurons, fluorescence intensity in the terminal was expressed as a percentage of the intensity in axon. Shown are the means $\pm S D(n=5$ or 6$)$. Asterisk indicates statistically significant difference ( $p \leq 0.01$; Student's $t$ test).
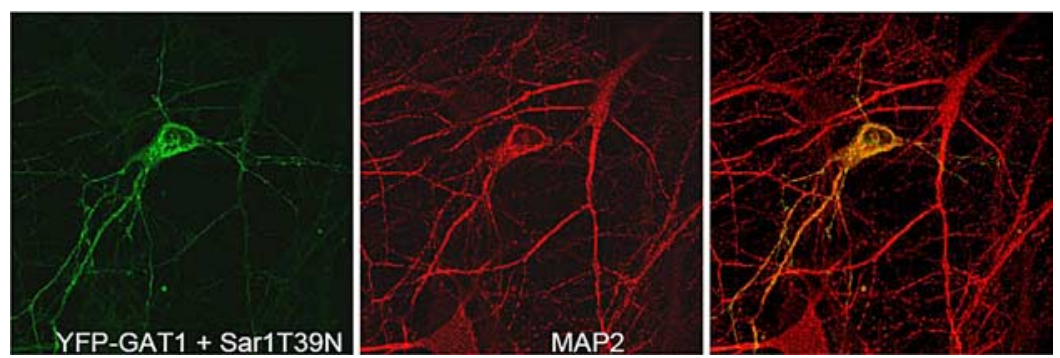

Figure 9. Localization of wild-type YFP-GAT1 in the absence of COPII function in neurons. Hippocampal neurons were prepared from rat embryos (E17). After $9 \mathrm{~d}$ in culture, neurons were transfected with plasmids encoding for YFP-tagged GAT1 and Sar1T39N. Cells were fixed after $24 \mathrm{~h}$ and immunostained for MAP2. Images were captured by confocal microscopy under settings that allow for visualization of YFP-tagged GAT1 (left panel) and MAP2 immunoreactivity (middle panel). The right panel was generated by overlaying the pictures. Data are representative of three independent transfections.

\section{ARFGAP1 controls trafficking of GAT1 in neurons}

It was of interest to document whether ARFGAP1 controls trafficking of (endogenous) GAT1 in neurons. We therefore overexpressed $\Delta 64$-ARFGAP1 and immunostained for GAT1 using the same antibody used for immunoprecipitations. This antibody has been used by others for immunohistochemical stainings of GAT1 (Manzano et al., 2007). GAT1 was expressed on the plasma membrane in cells that did not express $\Delta 64$-ARFGAP1 and was 
A
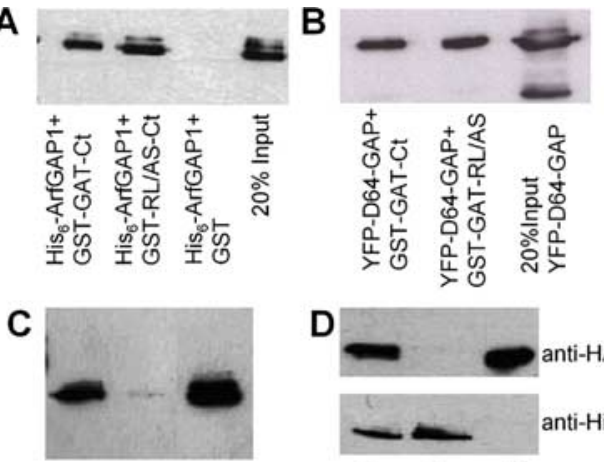

D

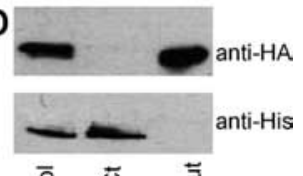

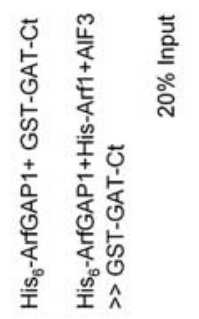

E

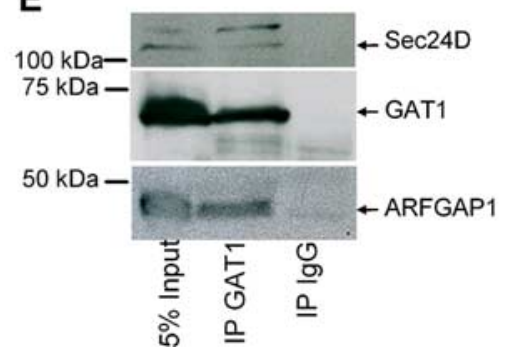

Figure 10. Interaction of ARFGAP1 with the $C$ terminus of GAT1. A, Purified GST-tagged $C$ terminus $(10 \mu \mathrm{g}$ ) of wild-type GAT1 (GST-GAT-Ct) or that of GAT1-RL/AS (GST-RLAS-Ct) or GST were incubated with His-tagged ARFGAP1 $(3 \mu \mathrm{g})$ for $2 \mathrm{~h}$ on ice. Subsequently, GSH-agarose was used to recover the proteins. $\boldsymbol{B}$, Cytosol (100 $\mu \mathrm{g}$ ) prepared from HEK293 cells overexpressing YFP-tagged $\triangle 64$ ARFGAP1 was incubated with GST-tagged C terminus of wild-type GAT1 (GSTGAT-Ct) or of GAT1-RL/AS (GST-RL/AS-Ct) for $2 \mathrm{~h}$ on ice followed by a GST-pulldown. C, GSTtagged C terminus $(10 \mu \mathrm{g}$ ) of GAT1 (GST-GAT-Ct) was incubated with His-tagged ARFGAP1 (4 $\mu \mathrm{g}$; His-ARFGAP1) for $2 \mathrm{~h}$ on ice (left lane) or His-ARFGAP1 was preincubated with $4 \mu \mathrm{g}$ of His-tagged Arf 1 in the presence of $\mathrm{AlF}_{3}$ for $30 \mathrm{~min}$ followed by $2 \mathrm{~h}$ incubation with GST-GAT1-Ct on ice (middle lane). GST-pulldown was performed, and ARFGAP1 was detected using an antiHis antibody. The right lane represents $20 \%$ of ARFGAP1 input used for the reaction. D, His ${ }_{6}$ tagged ARFGAP1 was incubated with cytosol (100 $\mu \mathrm{g})$ overexpressing HA-tagged Arf1 in the presence of $\mathrm{AlF}_{3}$ for $30 \mathrm{~min}$ (left lane) or $\mathrm{His}_{6}$-tagged ARFGAP1 was preincubated with GSTtagged GAT1 $\mathrm{C}$ terminus for $2 \mathrm{~h}$ before the reaction. Thereafter, $\mathrm{His}_{6}$-tagged ARFGAP1 was recovered using magnetic Ni-NTA beads. The amount of Arf1 that was pulled down was determined using anti-HA antibody (top blot). An antibody against the His ${ }_{6}$-tag was used to verify that the amount of ARFGAP1 retrieved by the beads was comparable (bottom blot). E, GAT1 was immunoprecipitated form adult rat brain as described in Materials and Methods. The right lane contains $5 \%$ input, the middle lane the eluate from the immunoprecipitation (IP) with an anti-GAT1 antibody (IP GAT1) and the left lane the mock immunoprecipitation with irrelevant $\lg G(I P \lg G)$. The numbers on the right side indicate the position of the molecular weight marker. Data are representative of three independent experiments.

found there in the soma and in one neurite at high density (Fig. $12 A)$. In cells expressing $\Delta 64$-ARFGAP1, GAT1 and $\Delta 64$ ARFGAP1 coaccumulated in punctate structures (Fig. 12 B). Together, these data show that ARFGAP1 controls neuronal trafficking of GAT1.

\section{Discussion}

In the current work, we used GAT1 as a model cargo to study the relevance of the early secretory pathway for sorting decisions of membrane proteins. There are several advantages of using GAT1.
One is that GAT1 must be delivered to the rim of the presynaptic specialization to allow for neuronal retrieval of synaptically released GABA. In fact, in mice engineered to express a fluorescent GAT1, presynaptic boutons of GABAergic interneurons in cerebellum and hippocampus have $800-1300$ GAT1 molecules/ $\mu \mathrm{m}^{2}$ and the axons that connect boutons have a linear density of 640 molecules/ $\mu \mathrm{m}$ (Chiu et al., 2002). Another advantage is that trafficking of GAT1 from ER to ERGIC (Scholze et al., 2002; Farhan et al., 2004, 2007) and from the ERGIC (Farhan et al., 2008) is reasonably well understood.

Recently, Ye et al. (2007) concluded from a mutational analysis of Drosophila neurons that dendritic growth required COPIIdependent ER export but that axonal growth was not disrupted by mutations of COPII components. In other words, the flow of lipids to support plasma membrane expansion is not dependent on COPII. However, it remains unclear whether protein trafficking to the axon requires the COPII machinery. Moreover the absence of the Golgi from axons (Horton et al., 2005; Ye et al., 2007) and polarization of the Golgi toward the major dendrite (Horton et al., 2005) raises the question whether sorting and targeting of proteins to the axon is dependent on the conventional secretory pathway. Here, we studied both, axonal delivery of the membrane cargo GAT1 and the relevance of the conventional secretory pathway for its correct axonal targeting.

We found that the Sec24-binding deficient mutant GAT1RL/AS reached the plasma membrane via an alternative nonconventional (i.e., COPII independent). This finding was important because it allowed us to compare GAT1-RL/AS with the wildtype transporter. We further characterized this pathway and found that it bypasses the ERGIC. The dependence of the wildtype GAT1 on COPII for ER export and its passage through the ERGIC gave us the opportunity to test whether transit through the conventional secretory pathway $\mathrm{ER} \rightarrow \mathrm{ERGIC} \rightarrow$ Golgi) determines correct axonal delivery of membrane cargo. Two alternative scenarios are conceivable: the first model posits that polarized sorting of membrane cargo is specified in the trans-Golgi network regardless of how the protein reaches the Golgi. In the alternative scenario, sorting decisions are contingent on correct transit through earlier steps, i.e., ER and/or ERGIC. Our data unequivocally show that wild-type GAT1 is transported along a COPIIdependent pathway from ER to ERGIC and that it must transit the ERGIC to be efficiently enriched in axon terminals. The failure of GAT1-RL/AS to be enriched in axon terminals clearly indicates that (1) COPII plays a role in delivering GAT1 to the axon terminal and (2) that early stations of the secretory pathway predetermine correct axonal delivery of membrane cargo.

As another remarkable finding, we report that ARFGAP1 interacts with GAT1 and is required for GAT1 trafficking. Originally, ARFGAP1 was identified by its eponymous action, i.e., triggering the GTPase of ARF1 (Cukierman et al., 1995) and thus driving the disassembly of the COPI coat by triggering the ARF1 GTPase. However, more recently, it has been appreciated that ARFGAP1 may play a role in earlier steps of vesicle budding: the yeast ARFGAP ortholog Glo3p was shown to be recruited to COPI-coated vesicles before ARF and to facilitate assembly of the COPI coat (Rein et al., 2002). In fact, mammalian ARFGAP1 apparently functions as a cargo adaptor that supports sorting of proteins into nascent COPI vesicles (Lee et al., 2005). Our observations are also consistent with a role of ARFGAP1 as a cargo adaptor: it mediates packaging of GAT1 into COPI vesicles that mediate anterograde transport from the ERGIC to the Golgi. Interference with exit of GAT1 from the ERGIC precluded its accumulation in neurites. This finding underscores the notion 
that early trafficking steps play a crucial role in determining proper targeting of GAT1.

Our observations imply that GAT1 must already recruit proteins required for sorting before reaching the Golgi. Earlier findings support this conjecture. The last three amino acids of GAT1 represent a type II-PDZ domain binding motif (McHugh et al., 2004). This motif puts GAT1 surface expression under the control of the exocyst which interacts with its C terminus (Farhan et al., 2004) (our unpublished observations). The exocyst catalyzes the very last stage in membrane protein trafficking, i.e., the fusion of the secretory vesicle with the cell surface. Nevertheless, if the interaction between GAT1 and the exocyst is disrupted, GAT1 does not accumulate in a submembranous compartment as would be expected, if only the insertion of secretory vesicles was impeded: in the absence of a functional exocyst, or upon mutation of the PDZ-motif, GAT1 is rather retained in the ER (Farhan et al., 2004). Thus, the exocyst must be recruited at an early stage. In fact, there is evidence that components of the exocysts reside on the ER membrane (Shin et al., 2000; Lipschutz et al., 2003; Guo and Novick, 2004). Finally, Muñiz et al. (2001) showed that different subtypes of COPII vesicles are generated from the ER, at least in vitro, and that these vesicles contain different types of cargo. This is a good precedent for sorting decisions made very early in the secretory pathway and importantly before the passage through the Golgi. It is highly likely that a similar distinctive sorting also operates at the level of the ER in neurons. Our observations suggest that membrane carriers required for axonal sorting of GAT1 are COPII-dependent. This COPII dependence sorts GAT1 into an anterograde pathway which assures its correct targeting. If GAT1 fails to enter this pathway, as in the case of GAT1-RL/AS, it does not become enriched in the axonal compartment.

Trafficking of membrane proteins through the secretory pathway is contingent on their sequential interaction with binding partners which allow for their recruitment into transport vesicles and for their correct delivery into the next compartment. Several proteins have been identified as binding partners of GAT1, with the potential to direct the route of GAT1 along the secretory pathway from the ER to its final destination, the presynaptic specialization. These include syntaxin-1 (Quick et al., 1997), GAT1 itself (Schmid et al., 2001), Pals-1
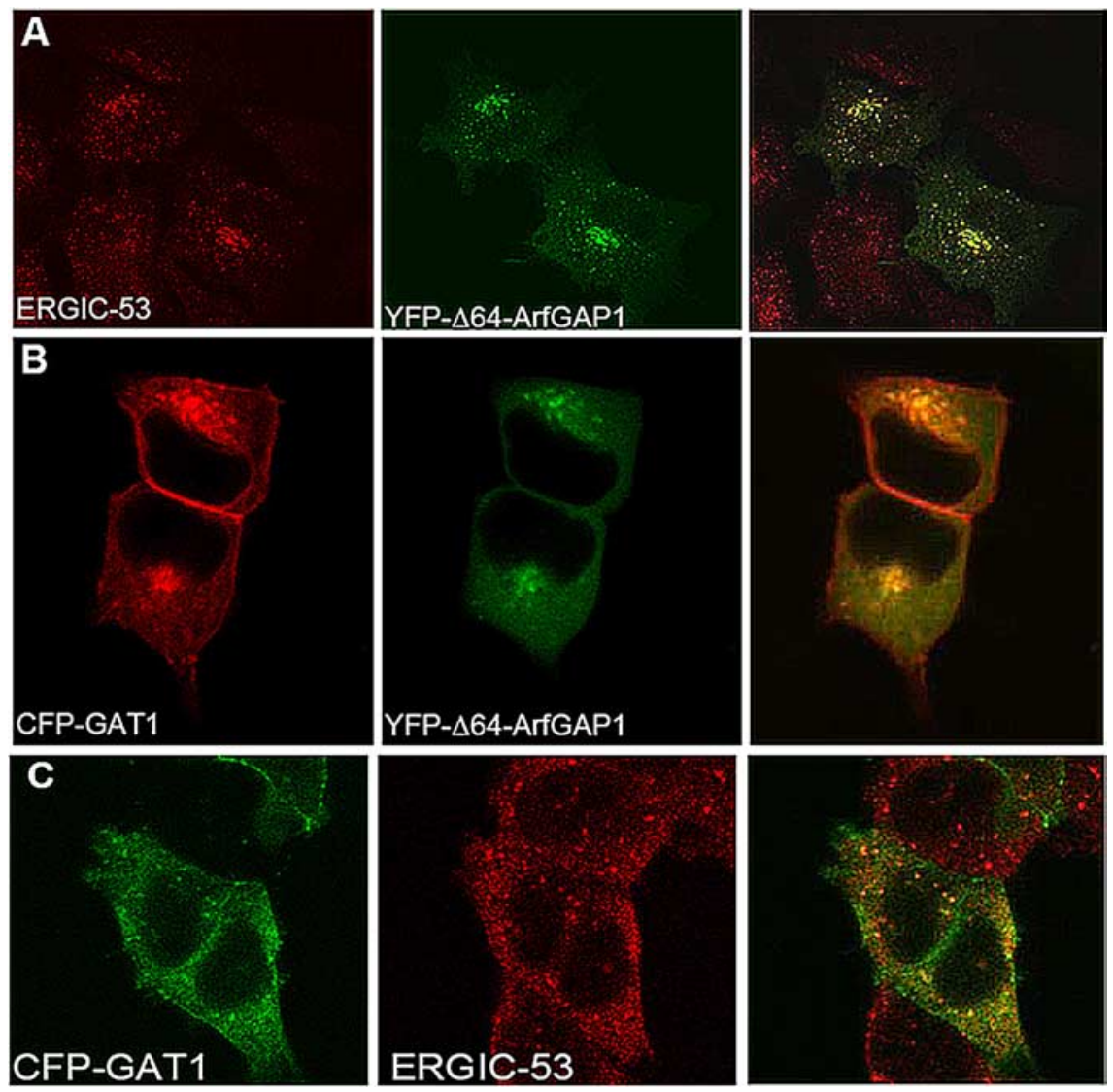

Figure 11. Effect of ARFGAP1 on subcellular localization of GAT1. A, HeLa cells were transfected with a plasmid encoding YFP-tagged $\triangle 64 A R F G A P 1$. On the next day, colocalization with ERGIC-53 was determined using immunofluorescence. $\boldsymbol{B}$, HEK293 cells were cotransfected with plasmids encoding CFP-tagged GAT1 and YFP-tagged $\triangle 64 A R F G A P 1$, and images were acquired $24 \mathrm{~h}$ later. C, HEK293 cells were transfected with a plasmid encoding CFP-tagged GAT1. On the next day, cells were treated with $50 \mu \mathrm{m}$ IC261. Cells were fixed with 4\% paraformaldehyde, and ERGIC-53 was detected by immunofluorescence. All images were acquired with a confocal microscope. The extent of colocalization was quantified in three independent experiments as outlined under Materials and Methods and in the legend to Figure 3 with 600/640 of ERGIC-53-containing vesicles (A) and 440/440 CFP-GAT1containing vesicles $(\boldsymbol{B})$ also containing YFP-tagged $\triangle 64 A R F G A P 1$ and 160/420 ERGIC-containing vesicles also containing YFPtagged $\triangle 64$ ARFGAP1.

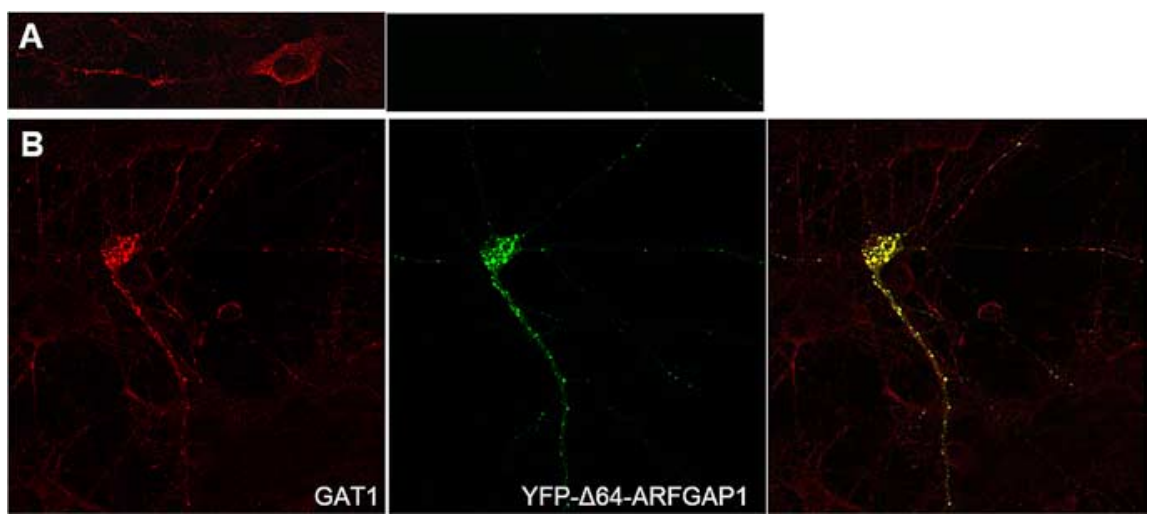

Figure 12. Effect of ARFGAP1 on subcellular localization of GAT1 in neurons. Hippocampal neurons were prepared from rat embryos (E17). After $9 \mathrm{~d}$ in culture, neurons were transfected with plasmids encoding for YFP-tagged $\Delta 64$ ARFGAP1. Cells were fixed after $48 \mathrm{~h}$ and immunostained for GAT1. Images were captured by confocal microscopy. A shows immunostaining for GAT1 (left panel) in a neuron that underwent the transfection procedure but did not express YFP-tagged $\triangle 64 A R F G A P 1$ (right panel). $\boldsymbol{B}$ shows staining for GAT1 (left panel) in a neuron expressing YFP-tagged $\triangle 64 A R F G A P 1$ (middle panel) and the resulting overlay (right panel). The extent of colocalization was quantified in three independent experiments as outlined under Materials and Methods and in the legend to Figure 3 with 111/136 of YFP-tagged $\triangle 64$ ARFGAP1 vesicles also containing GAT1. 
(McHugh et al., 2004), and Sec24D (Farhan et al., 2004, 2007). While syntaxin- 1 and Pals- 1 may be important in the final stage, i.e., the accumulation of GAT1 at the synapse, selfassembly into GAT1 oligomers (Farhan et al., 2006) and interaction with Sec24D are required for the very first step of COPII-dependent export of GAT1 from the ER (Farhan et al., 2007). Here, we identified ARFGAP1 as an additional interaction partner of GAT1. Our experiments showed that ARFGAP1 was required to support trafficking of GAT1 from the ERGIC. There are many arguments derived from live cell imaging in support of the notion that the ERGIC is a stationary compartment (Ben-Tekaya et al., 2005). Moreover, a mutation can be introduced into the GAT1 C terminus that results in selective retention of the resulting GAT1-SSS in the ERGIC (Farhan et al., 2008). Thus, anterograde carriers must exist that transfer cargo, such as GAT1, from ERGIC to Golgi. Our finding that ARFGAP1 can act as a cargo adaptor for GAT1 is therefore consistent with a process of regulated sorting of proteins at the level of the ERGIC and a role of COPI in this process (Shima et al., 1999).

In summary, the different mutants of GAT1 enabled us to characterize its trafficking in the secretory pathway. In addition they allowed us to test the dependence of axonal sorting on early secretory events. Our results clearly show that COPII-mediated ER export is crucial for correct targeting of GAT1 to the axon terminal.

\section{References}

Appenzeller-Herzog C, Hauri HP (2006) The ER-Golgi intermediate compartment (ERGIC): in search of its identity and function. J Cell Sci 119:2173-2183.

Ben-Tekaya H, Miura K, Pepperkok R, Hauri HP (2005) Live imaging of bidirectional traffic from the ERGIC. J Cell Sci 118:357-367.

Chiu CS, Jensen K, Sokolova I, Wang D, Li M, Deshpande P, Davidson N, Mody I, Quick MW, Quake SR, Lester HA (2002) Number, density, and surface/cytoplasmic distribution of GABA transporters at presynaptic structures of knock-in mice carrying GABA transporter subtype 1-green fluorescent protein fusions. J Neurosci 22:10251-10266.

Cukierman E, Huber I, Rotman M, Cassel D (1995) The ARF1 GTPaseactivating protein: zinc finger motif and Golgi complex localization. Science 270:1999-2002.

Farhan H, Korkhov VM, Paulitschke V, Dorostkar MM, Scholze P, Kudlacek O, Freissmuth M, Sitte HH (2004) Two discontinuous segments in the carboxyl terminus are required for membrane targeting of the rat gammaaminobutyric acid transporter-1 (GAT1). J Biol Chem 279:28553-28563.

Farhan H, Freissmuth M, Sitte HH (2006) Oligomerization of neurotransmitter transporters: a ticket from the endoplasmic reticulum to the plasma membrane. Handb Exp Pharmacol 175:233-249.

Farhan H, Reiterer V, Korkhov VM, Schmid JA, Freissmuth M, Sitte HH (2007) Concentrative export from the endoplasmic reticulum of the gamma-aminobutyric acid transporter 1 requires binding to SEC24D. J Biol Chem 282:7679-7689.

Farhan H, Reiterer V, Kriz A, Hauri H-P, Pavelka M, Sitte HH, Freissmuth M (2008) Signal-dependent export of the GABA transporter-1 from the intermediate compartment is specified by a carboxyl terminal motif. J Cell Sci 121:753-761.

Gomez M, Scales SJ, Kreis TE, Perez F (2000) Membrane recruitment of coatomer and binding to dilysine signals are separate events. J Biol Chem 275:29162-29169.

Griffiths G, Pfeiffer S, Simons K, Matlin K (1985) Exit of newly synthesized membrane proteins from the trans cisterna of the Golgi complex to the plasma membrane. J Cell Biol 101:949-964.

Guo Q, Vasile E, Krieger M (1994) Disruptions in Golgi structure and membrane traffic in a conditional lethal mammalian cell mutant are corrected by epsilon-COP. J Cell Biol 125:1213-1224.

Guo W, Novick P (2004) The exocyst meets the translocon: a regulatory circuit for secretion and protein synthesis? Trends Cell Biol 14:61-63.

Hauri HP, Kappeler F, Andersson H, Appenzeller C (2000) ERGIC-53 and traffic in the secretory pathway. J Cell Sci 113:587-596.
Horton AC, Rácz B, Monson EE, Lin AL, Weinberg RJ, Ehlers MD (2005) Polarized secretory trafficking directs cargo for asymmetric dendrite growth and morphogenesis. Neuron 48:757-771.

Huber I, Cukierman E, Rotman M, Aoe T, Hsu VW, Cassel D (1998) Requirement for both the amino-terminal catalytic domain and a noncatalytic domain for in vivo activity of ADP-ribosylation factor GTPaseactivating protein. J Biol Chem 273:24786-24791.

Krijnse-Locker J, Parton RG, Fuller SD, Griffiths G, Dotti CG (1995) The organization of the endoplasmic reticulum and the intermediate compartment in cultured rat hippocampal neurons. Mol Biol Cell 6:1315-1332.

Lee MC, Miller EA, Goldberg J, Orci L, Schekman R (2004) Bi-directional protein transport between the ER and Golgi. Annu Rev Cell Dev Biol 20:87-123.

Lee SY, Yang JS, Hong W, Premont RT, Hsu VW (2005) ARFGAP1 plays a central role in coupling COPI cargo sorting with vesicle formation. J Cell Biol 168:281-290.

Li Y, Hou LX, Aktiv A, Dahlström A (2007) Studies of the central nervous system-derived CAD cell line, a suitable model for intraneuronal transport studies? J Neurosci Res 85:2601-2609.

Lipschutz JH, Lingappa VR, Mostov KE (2003) The exocyst affects protein synthesis by acting on the translocation machinery of the endoplasmic reticulum. J Biol Chem 278:20954-20960.

Manzano J, Cuadrado M, Morte B, Bernal J (2007) Influence of thyroid hormone and thyroid hormone receptors in the generation of cerebellar $\gamma$-aminobutyric acid-ergic interneurons from precursor cells. Endocrinology 148:5746-5751.

McHugh EM, Zhu W, Milgram S, Mager S (2004) The GABA transporter GAT1 and the MAGUK protein Pals1: interaction, uptake modulation, and coexpression in the brain. Mol Cell Neurosci 26:406-417.

Muñiz M, Morsomme P, Riezman H (2001) Protein sorting upon exit from the endoplasmic reticulum. Cell 104:313-320.

Nickel W, Malsam J, Gorgas K, Ravazzola M, Jenne N, Helms JB, Wieland FT (1998) Uptake by COPI-coated vesicles of both anterograde and retrograde cargo is inhibited by GTP $\gamma \mathrm{S}$ in vitro. J Cell Sci 111:3081-3090.

Pepperkok R, Scheel J, Horstmann H, Hauri HP, Griffiths G, Kreis TE (1993) Beta-COP is essential for biosynthetic membrane transport from the endoplasmic reticulum to the Golgi complex in vivo. Cell 74:71-82.

Pepperkok R, Whitney JA, Gomez M, Kreis TE (2000) COPI vesicles accumulating in the presence of a GTP restricted arf1 mutant are depleted of anterograde and retrograde cargo. J Cell Sci 113:134-144.

Pierce JP, van Leyen K, McCarthy JB (2000) Translocation machinery for synthesis of integral membrane and secretory proteins in dendritic spines. Nat Neurosci 3:311-313.

Qi Y, Wang JK, McMillian M, Chikaraishi DM (1997) Characterization of a CNS cell line, CAD, in which morphological differentiation is initiated by serum deprivation. J Neurosci 17:1217-1225.

Quick MW, Corey JL, Davidson N, Lester HA (1997) Second messengers, trafficking-related proteins, and amino acid residues that contribute to the functional regulation of the rat brain GABA transporter GAT1. J Neurosci 17:2967-2979.

Rein U, Andag U, Duden R, Schmitt HD, Spang A (2002) ARF-GAPmediated interaction between the ER-Golgi v-SNAREs and the COPI coat. J Cell Biol 157:395-404.

Sannerud R, Marie M, Nizak C, Dale HA, Pernet-Gallay K, Perez F, Goud B, Saraste J (2006) Rabl defines a novel pathway connecting the pre-Golgi intermediate compartment with the cell periphery. Mol Biol Cell 17:1514-1526.

Sans N, Wang PY, Du Q, Petralia RS, Wang YX, Nakka S, Blumer JB, Macara IG, Wenthold RJ (2005) mPins modulates PSD-95 and SAP102 trafficking and influences NMDA receptor surface expression. Nat Cell Biol 7:1179-1190.

Saraste J, Kuismanen E (1984) Pre- and post-Golgi vacuoles operate in the transport of Semliki forest virus membrane glycoproteins to the cell surface. Cell 38:535-549.

Scheffzek K, Ahmadian MR, Kabsch W, Wiesmüller L, Lautwein A, Schmitz F, Wittinghofer A (1997) The Ras-RasGAP complex: structural basis for GTPase activation and its loss in oncogenic Ras mutants. Science 277:333-338.

Schmid JA, Scholze P, Kudlacek O, Freissmuth M, Singer EA, Sitte HH (2001) Oligomerization of the human serotonin transporter and of the 
rat GABA transporter 1 visualized by fluorescence resonance energy transfer microscopy in living cells. J Biol Chem 276:3805-3810.

Scholze P, Freissmuth M, Sitte HH (2002) Mutations within an intramembrane leucine heptad repeat disrupt oligomer formation of the rat GABA transporter 1. J Biol Chem 277:43682-43690.

Schweizer A, Fransen JA, Matter K, Kreis TE, Ginsel L, Hauri HP (1990) Identification of an intermediate compartment involved in protein transport from endoplasmic reticulum to Golgi apparatus. Eur J Cell Biol 53:185-196.

Shima DT, Scales SJ, Kreis TE, Pepperkok R (1999) Segregation of COPIrich and anterograde-cargo-rich domains in endoplasmic-reticulum-toGolgi transport complexes. Curr Biol 9:821-824.

Shin DM, Zhao XS, Zeng W, Mozhayeva M, Muallem S (2000) The mam- malian Sec6/8 complex interacts with $\mathrm{Ca}(2+)$ signaling complexes and regulates their activity. J Cell Biol 150:1101-1112.

Wendeler MW, Paccaud JP, Hauri HP (2007) Role of Sec24 isoforms in selective export of membrane proteins from the endoplasmic reticulum. EMBO Rep 8:258-264.

Xu D, Hay JC (2004) Reconstitution of COPII vesicle fusion to generate a pre-Golgi intermediate compartment. J Cell Biol 167:997-1003.

Ye B, Zhang Y, Song W, Younger SH, Jan LY, Jan YN (2007) Growing dendrites and axons differ in their reliance on the secretory pathway. Cell 130:717-729.

Yu S, Roth MG (2002) Casein kinase I regulates membrane binding by ARF GAP1. Mol Biol Cell 13:2559-2570. 